\author{
Classification \\ Physics Abstracts \\ 02.10.Gd
}

\title{
Use of Mathematical Morphology in Industrial Control
}

\author{
Jean Serra \\ CMM, École des Mines de Paris, 35 rue Saint-Honoré, 77305 Fontainebleau Cedex, France
}

Résumé. - En vision par ordinateur, la pertinence de la démarche est fondée sur un certain nombre de critères. On en étudie cinq ici, à savoir le mode de représentation, l'éventuelle présence d'individus, l'invariance par translation, les mesures et la vitesse. Ces critères ne sont pas présentés dans l'abstrait, mais discutés, en situation, au moyen de cinq exemples qui les mettent préférentiellement en valeur.

\begin{abstract}
Computer vision is used in several types of industrial controls. The relevance of such an approach involves a certain number of criteria. Five of them are investigated here, namely the mode of representation, the possible presence of individual objects, translation invariance, measurements and speed. These criteria are not analysed in abstracto, but rather in situation, i.e. via five examples. Each example emphasizes some criterion or other more specifically.
\end{abstract}

\section{Five Criteria}

1.1 The Representation. - Generally speaking, the "image", in image processing, is a certain representation of the real world, and not this world itself. This representation strongly depends on the size of the object under study, on its magnification (two maps of a same region, at two different scales provide different information), on its light, on its sampling (compare the sections of a human abdomen by scanner, with its projection by X-ray photograph), and on its possible coloration or staining. In terms of industrial control, this means that we must build the object of study, or re-build it, from the real world. This reconstruction is, of course, submitted to some constraints, but the way to do it is rarely unique.

Our first example, about size control of glass fibers, illustrates this point [5]. One can either lay down the fibers or embed them in resin and make cross sections, as shown in Figure 1. What is the best way?

1.2 INDIVIDUALS. - The second criterion is associated with the possible existence of particles, or more generally of individuals, i.e. of subparts of the image that are considered as wholes (e.g. a city, seen from aerial photograph, or a mitosis, in chromosome analysis, are (disconnected) individuals). In such cases, the processing necessarily begins on the complete image: a thresholding, for example, works at the level of the pixel, and ignores the individuals. Then, the question arises 
a)

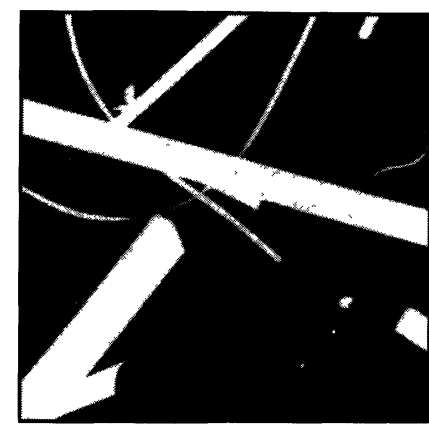

b)

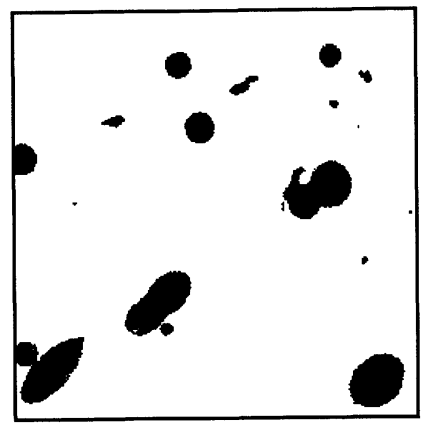

Fig. 1. - Example $n^{0}$ 1: glass fibers. a) Electron micrograph. b) Optical micrograph from a cross section of specimen a.

to know when to shift to individuals processing (if need be...). The rice example (No 2), where the proportion of broken grains is asked, or the motor example (Sect. 4), where the presence of a has to be checked, illustrate this situation.

1.3 TRANSLATION INVARIANCE. - In a sense, the second criterion may appear as a particular case of a third, and more subtle one, namely the opportunity to consider the scene under study as invariant under translation. The radial vannes whose presence is checked in example No 5 occupy a very characteristic place in the field of view, it should be a pity not to exploit this $a$ priori information. Moreover, we find the grains of rice, which are uniformly displayed, and, in between, the antibiograms of example No 3, which are roughly periodic. To which extent do we have to leave the comfortable world of translation invariant algorithms when studying such cases?

1.4 Metrology. - The example of the antibiograms, that we have just encountered, is instructive also in other respects. The background layer is generated by a microbial culture which developed uniformly. A few pastilles of different antibiotics have then been put on the culture and have more or less killed the bacteria around them. In other words, the radii of the dark circular crowns increase with the activity of the antibiotics dropped in their middle. How to measure these radii? Such problem of metrology depends indeed on another question, namely: for the final diagnosis, what accuracy is really needed? (Clearly the robustness of the processing, and the correction of possible bias depend on the answer).

1.5 SpeED. - The last criterion deals with the processing time. What speed is requested, or required? The confused notion of "real time", often referred to here, does not say what "reality" is meant. In case of antibiograms, the speed of the phenomenon is that of the microbiological reaction, i.e. a few hours. In case of engine inspection, the speed becomes that of the production line, i.e. a few seconds between the apparition of two successive engines in the axis of the camera. In both cases, the relative simplicity of the processing allows to ignore the speed constraint. But other situations are more drastic, such as the reading of addresses on envelopes. A high flow of data combined with a complex series of algorithms, lead to a search for software optimization, and finally to the design of specific hardwares.

The four examples which follow illustrate one criterion each, at least. The morphological operators they involve are indicated, and their effects are shown on photographs. For a more formal description of these operations the reader may refer to [2] or to [3]. 


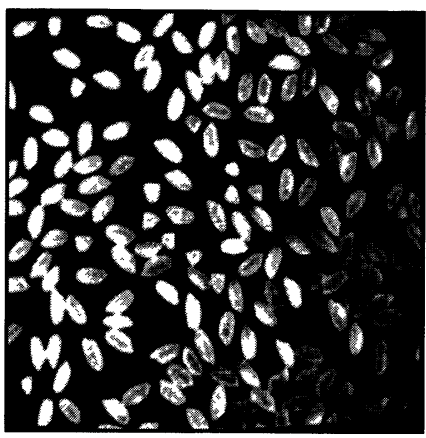

a)

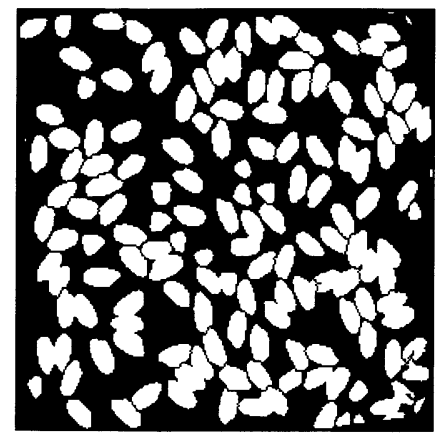

b)

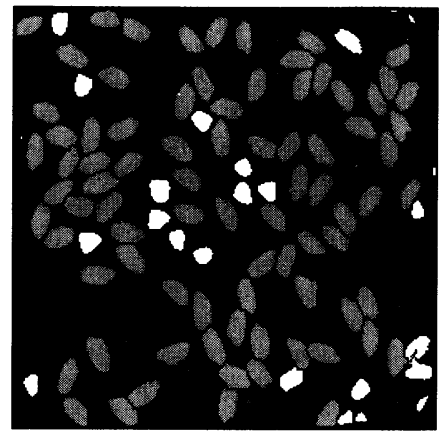

c)

Fig. 2. - Example $\mathrm{n}^{\circ}$ 2: broken rice grains. a) Initial image. b) Segmentation of a. c) Grains which are integer (grey); broken (white); clustered (dark).

\section{Rice Quality and Individual Analysis}

This example illustrates the two criteria about individuals and about metrology. A hundred or so of rice grains are spread out for T.V. inspection (Fig. 2a). It is asked to determine by image analysis, the proportion of broken grains. One may proceed as follows:

a) binarization of the input image, by means of the top-hat transform, followed by a thresholding;

b) a number of clusters appear on the binary image, they will be partially reduced by shape segmentation based on the watershed of the distance function. This results in Figure $2 b$;

c) the particles that hit the edges of the field do not lend themselves to measurements, they must be removed;

d) the connected components of set $2 \mathrm{c}$ are sorted out into the three categories of "clusters", "integer grains" and "broken grains", according to their areas (Fig. 2c).

Comments. The notion of a particle appears in the algorithms at step d only. The pre-treatments $\mathrm{b}$ and $\mathrm{c}$ hold on a set where individual objects are not differentiated. Alternately, we could have isolated them at the very beginning of the process. It would have allowed more sophisticated treatments, but for a longer computational time. Similarly, the decision to binarize the input may be called into question.

The choice adopted here is to accept clusters, assuming that they are correctly sorted out at the last step. By so doing, we deliberately introduce a bias, since the broken grains are less present in the clusters than the integer ones. Of course, but the elimination of the edge cutting grains also introduces a bias, and the way the handful of rice is taken from the sack brings into play a third, and worse, bias. All of them pertain to a certain sampling scheme, that must be approached experimentally. In brief, this example shows that individual analysis often leads to sampling problems, and metrology to bias.

\section{Antibiograms and Robust Metrology}

This second study deals with robustness, metrology and possible lack of translation invariance. As we saw in Section 1.4, the task of the processing is to calculate the radii of the dark halos which 


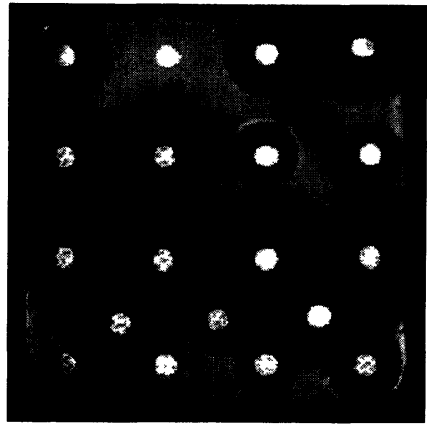

a)

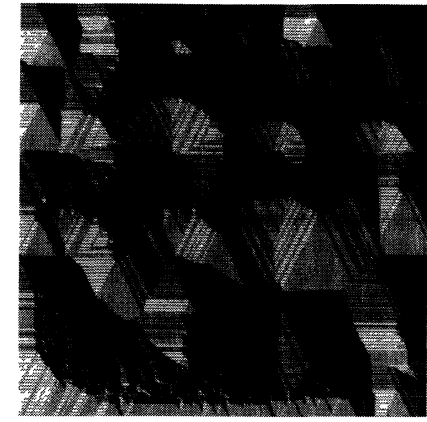

b)

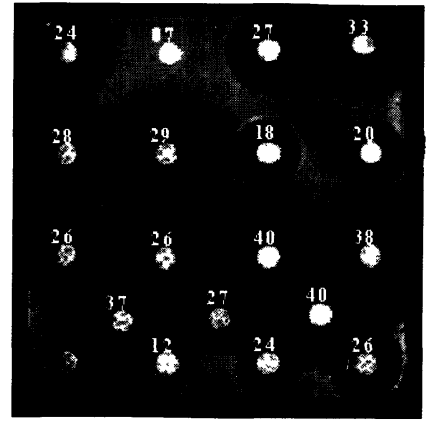

c)

Fig. 3. - Example ${ }^{0}$ 3: antibiograms. a) Initial image. b) Distance function. c) Radii of the halos.

partly overlap (Fig. 3a), and which delineate the zones of action of the antibiotics located at their centers (white pastilles). Here, the processing steps are the following:

a) the set of the pastilles are obtained by thresholding the residual of a morphological filter by reconstruction;

b) a "raw" threshold of the initial image (Fig. 3a) generates a set whose distance function is computed (Fig. 3b);

c) the maximum value of this distance function, taken in each pastille gives the radius of the corresponding halo (Fig. 3c).

Comments. The approach described in step b has been chosen for the sake of robustness. As a matter of fact, the intensity of the bacteria culture varies from place to place, and from box to box. But moreover, when an antibiotic acts, it cleans the area around it by leaving a uniform dark zone. So when thresholding between 0 and $t$ the 8-bit input image, the core of each halo is robust under small variations of $t$. Moreover, the distance function, which is an upper semi-continuous mapping, preserves such a robustness. Hence there are neither false negative, nor false positive, and the most efficient pastilles are detected. The therapeutic purposes do not demand more.

\section{Engines and Translation Invariance}

This example derives from Sternberg's work [4], with some changes. Oil pumps for car engines file past the axis of a vision unit. Its processing must check whether all vannes are in place. Figures $4 \mathrm{a}$ and $4 \mathrm{~b}$ represent a positive and a negative cases respectively. The idea of the processing is to play with the (quasi) radial symmetry of the pump, so to isolate the region of interest. This leads to the following steps:

a) by means of an alternating filter by reconstruction, and a threshold, produce a binary version of the clear zones. This results in the dark grey set of Figure 4c;

b) we know that the centre of the field (i.e. the white central point), must fall in the central circular pore. This marker allows to extract the pore, and, from the pore, the set connected component which is adjacent to it. The result is indicated in white in Figure 4c;

c) if all seven vannes are present, this white particle must exhibit seven holes (Fig. 4d), if not, it has less holes (e.g. 5 pores in Fig. 4c). 


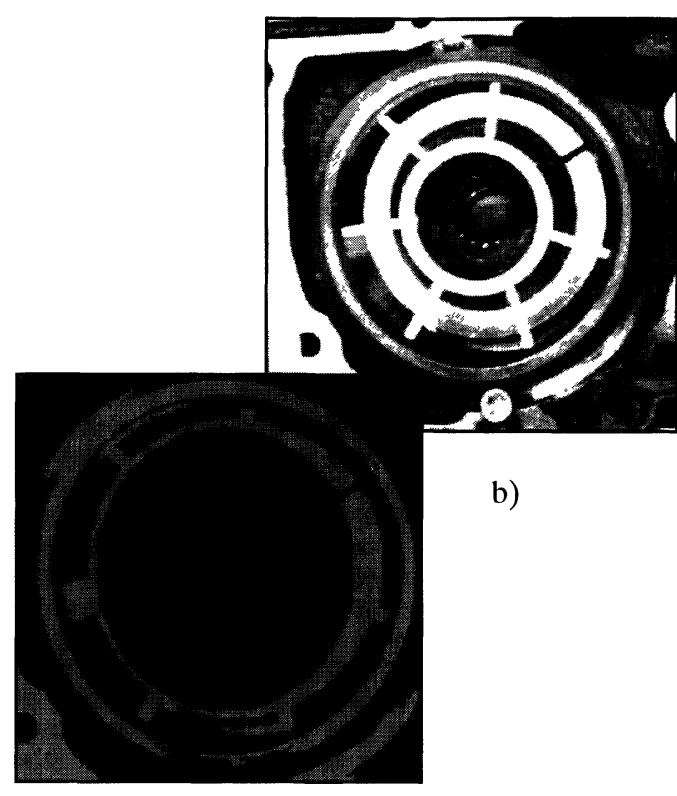

a)

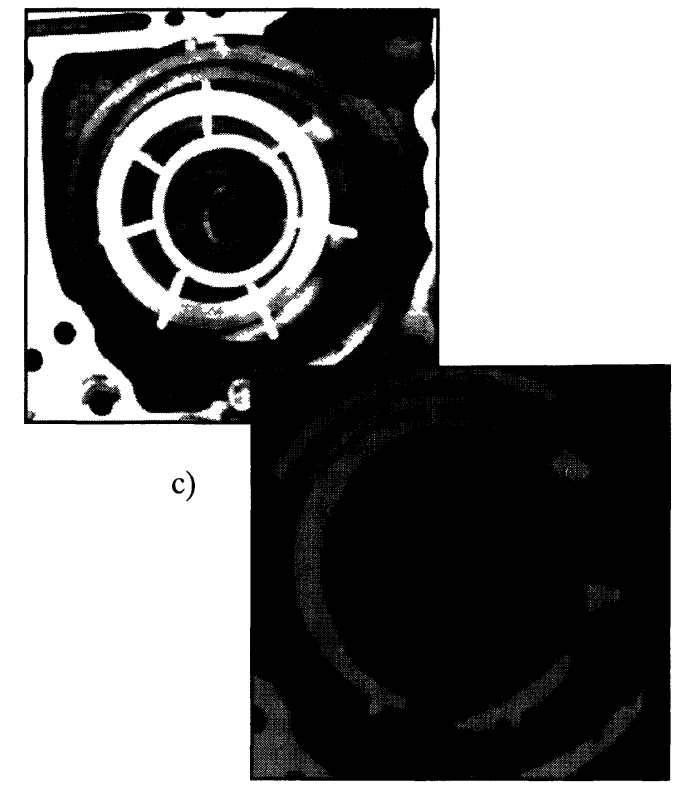

d)

Fig. 4. - Example $\mathrm{n}^{0}$ 4: oil pump inspection. a, b) Initial images in a positive and a negative cases. c, d) Grey: first selection; white, central point and zone whose connectivity has to be measured.

Comments. Without the precious indication of the center location, the count would have been much more difficult, and risky. Note that we moved away from translation invariance by introducing one isolated point in the scene under study. The way the loss of information has been managed is also instructive. The initial filter on the greytone image was idempotent (a closing followed by an opening), the threshold which follows is still idempotent, and the third operation, namely the white particle extraction from the central marker, is again idempotent (but not increasing).

\section{Addresses on Envelopes and Speed}

Today, there exist excellent techniques to recognize printed characters, when they are correctly proposed to a vision sensor. The trouble comes with the automatic extraction of the zone to look at. In case of addresses on envelopes, the presence of plastic covers, which multiply parasitic reflections, and of an accumulation of drawings and symbols (Fig. 5a) do not simplify the task. The study we report here was performed by Beucher et al. in 1995 [1] and is currently tested by the French Post Administration. It illustrates typically the conflict between speed and accuracy.

Ninety per cent of the letters are printed matter, and need a $2000 \times 2000$ digitization. The address blocks use dark fonts on a clear background, and their characters turns out to be small isolated objects, regularly aligned in some particular directions. It is this set of features which enables their extraction. One may proceed as follows:

a) infimum of the geodesic dilations in 44 directions by long segments;

b) an alternated filter performed on the previous infimum leads to Figure 5 b; by thresholding this set according to the depth of the minima, one generates a mask which extracts the regions of interest (Fig. 5c). The latter are then transmitted to the automatic reading units. 


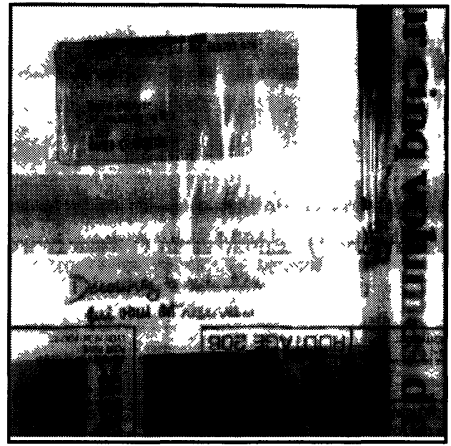

a)

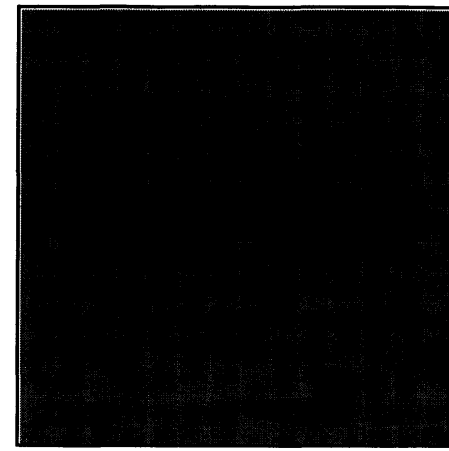

b)

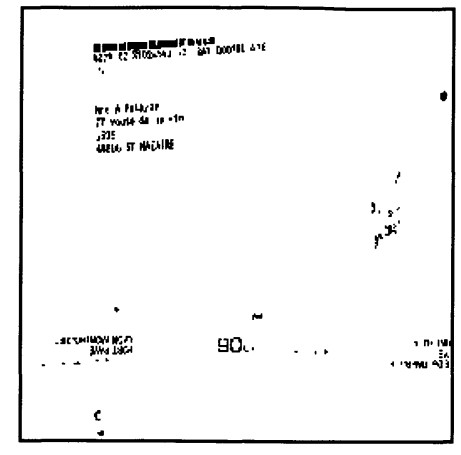

c)

Fig. 5. - Example $n^{\circ}$ 5: addresses reading. a) Initial image. b) Filtered version of a. c) Regions of interest.

Comments. Although an inf operator may be parallelized, step a, when applied to huge images is necessarily time consuming. An alternative processing replaces it either by an inf over 8 directions only, or even by a unique isotropic geodesic dilation of small size: the filtering holds on the thickness of the dark regions instead of their lengths. The results are comparatively less accurate but may suffice for downstream treatments. Only routine experiments, and improvements in the downstream character recognition will lead to an optimal solution.

\section{Conclusion}

In conclusion, we would like to emphasize two features which appear systematically in industrial control. It is less a matter of technical criterion than of management, but they do not concern profitability, which is contemplated here.

First a distinction has to be established between on-line control, and indirect controls in laboratories, when experimental protocols attempt to find the key parameters. The first ones are not always depending on the second ones, which aim basically to understand what is going on. Also, on-line controls require global optimizations of the various processes along the line (e.g. envelopes control), whereas the laboratory approach focuses on some steps specifically. However (and this is the second feature), in both cases, the situation is clearly defined only when the industrialist or the physicist on the one hand, and the specialist in vision on the other hand, agree on a common data base for representing the phenomenon under study. We know, by personal experience, that when control by computerized vision fails, it is most often because the two parties have avoided to agree on a set of representative images.

\section{References}

[1] Beucher S., Kozyrev S. and Gorokhovik D., Pré-traitement morphologique d'images de plis postaux (CNED'96, Nantes).

[2] Heijmans H.J.A.M., Morphological Image Operators (London: Ac. Press, 1994).

[3] Serra J., Image Analysis and Mathematical Morphology, Vol. I (London: Ac. Press, 1982); J. Serra, Ed., Image Analysis and Mathematical Morphology, Vol. II, Theoretical Advances (London: Ac. Press, 1988).

[4] Sternberg S.R., Morphology for grey tone functions, CVGIP 35 (1986) 333-355.

[5] Talbot H., Analyse morphologique de fibres minérales d'isolation, Thèse de Doctorat de l'École des Mines de Paris (1993). 